\title{
Field Quality Optimization in a Common Coil Magnet Design*
}

\author{
Ramesh Gupta and Suitbert Ramberger \\ Lawrence Berkeley National Laboratory, Berkeley, CA 94720 USA \\ Stephan Russenschuck \\ CERN, Geneva, Switzerland
}

\begin{abstract}
This paper presents the results of initial field quality optimization of body and end harmonics in a "common coil magnet design" [1-6]. It is shown that a good field quality, as required in accelerator magnets, can be obtained by distributing conductor blocks in such a way that they simulate an elliptical coil geometry. This strategy assures that the amount of conductor used in this block design is similar to that is used in a conventional cosine theta design. An optimized yoke that keeps all harmonics small over the entire range of operation using a single power supply is also presented. The field harmonics are primarily optimized with the computer program ROXIE [7,8].
\end{abstract}

\section{INTRODUCTION}

The common coil magnet design has a potential of significantly reducing the cost of the proposed Very Large Hadron Collider (VLHC) [9] based on high field magnet option. The common coil design is a block-type 2-in-1 magnet design where the main coils are shared between the two apertures in a top-bottom configuration. The concept has been recently extended to a 4-in-1 magnet design [2]. One half of the 40 $\mathrm{mm}$ aperture magnet coldmass is shown in Fig. 1. The computed quench field at $4.2 \mathrm{~K}$ is $14.8 \mathrm{~T}$ with $\mathrm{Nb}_{3} \mathrm{Sn}$ conductor. Major parameters of this design are given in section III (Table III).

In past a major concern against using a block coil geometry in a high field accelerator magnet was a perception that a good quality can not be obtained using a similar amount of conductor that is used in cosine theta design. However, the work presented here shows the typical field quality requirement of accelerator dipoles (field harmonics $<10^{-5}$ ) can be achieved in this design. Likewise, McIntyre, et al. [10] show a good field quality in an another type of high field block dipole design.

The field quality is expressed in terms of the normal and skew harmonics, $b_{n}$ and $a_{n}$, defined in the following expansion of horizontal and vertical components of field $\left(B_{x}, B_{y}\right)$ :

$$
B_{y}+i B_{x}=10^{-4} \times B_{1} \sum_{n=1}^{\infty}\left(b_{n}+i a_{n}\right)[(x+i y) / R]^{n-1}
$$

where $x$ and $y$ are the horizontal and vertical coordinates, $B_{1}$ is the dipole field strength at $x=R$ and $R$ is the "reference radius" that is chosen to be $10 \mathrm{~mm}$ here. The values of the field harmonic are given in units of $10^{-4}$.

Manuscript received on September 27, 1999. Revised on November 5, 1999. R. Gupta, Email : RGupta@lbl.gov, FAX 510-486-5310.

Work supported by the U.S. Department of Energy under the contract No. DEAC03-76SF00098.

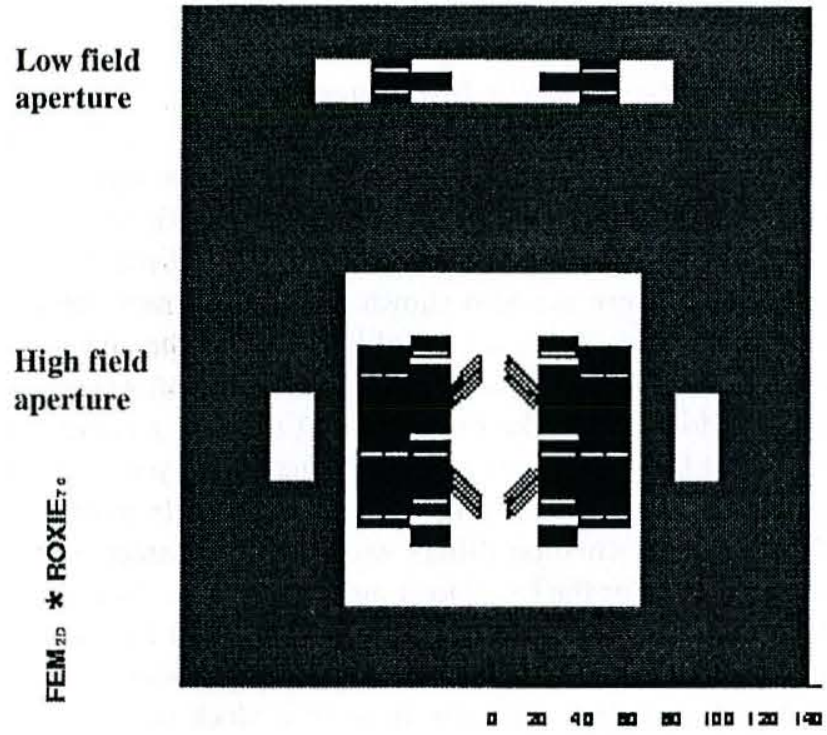

Fig 1. Upper half of the 4-in-1 common coil magnet system (see Fig. 4 for an end-view of the full model).

\section{OPTIMIZATION OF FIELD HARMONICS}

Field harmonics in the magnet body (straight section) are minimized with the help of spacers (equivalent to wedges in cosine theta magnets) and the parameters of the "auxiliary coils". An auxiliary coil refers to the partial layer near the vertical axis that typically contains five turns. In an actual magnet structure the auxiliary coil, particularly the lower one of the upper high field aperture (see Fig. 1), may become a part of the main coil. In the ends of the magnet, the turns of the auxiliary coils bend vertically downwards or upwards away from the magnet bore to allow for a large bend radius.

Conceptually the coil is minimized to produce an elliptical geometry. However, in reality after specifying the initial configuration, the computer code ROXIE optimizes the parameters within the specified constraints to generate whatever geometry that gives low field harmonics. One issue of concern from the mechanical structure standpoint is the auxiliary coil with tilted blocks of conductor. For this reason, several configurations (cases) for the auxiliary coils are examined and some of them contain no tilted blocks. These different configurations of auxiliary coils can be tested individually in a magnet where the main coils remain the same. The result of these tests may help develop a design that is optimum in an overall sense. 
The following sub-sections describe the overall field quality optimization. An example of this optimization process is presented. Since the field quality optimization has only recently been started, the results should not be viewed as the ultimate field quality that can be expected in a magnet design based on the common coil geometry. Nevertheless, the results are sufficient to prove that the field quality needed in accelerator magnets can be obtained.

\section{A. Magnet Body-Geometric Harmonics}

Figure 2 shows a number of possibilities (cases) for auxiliary coil geometry that are being currently examined for a field quality magnetic design. The main coils and a part of the iron yoke structure are also shown together. Except for the case No. 1, the cable used in auxiliary coils is the same as in the innermost layer of the main coil. The two outermost layers form a double pancake racetrack coil and use a cable that is different from the one used in the innermost layer.

The cables are bent the easy way for the main coils in all cases. However, the situation differs widely for the cables in the auxiliary coils. For the case No. 1 and 2, the cables in the ends of the auxiliary coils are bent the easy way and for the case No. 3, the cables in the ends are bent the hard way. For the case No. 4 , auxiliary coils are made of a block of turns that are tilted. The ends of this auxiliary coil may therefore be wound the same way as that in cosine theta magnets. However, the situation might be better as compared to the cosine theta magnets as the bend radius in the ends of the common coil design is an order of magnitude larger than that in the conventional cosine theta or conventional block dipole designs. It is due to the fact that in the common coil ends, the bend radius is determined by the separation between two apertures rather than the size of aperture itself.
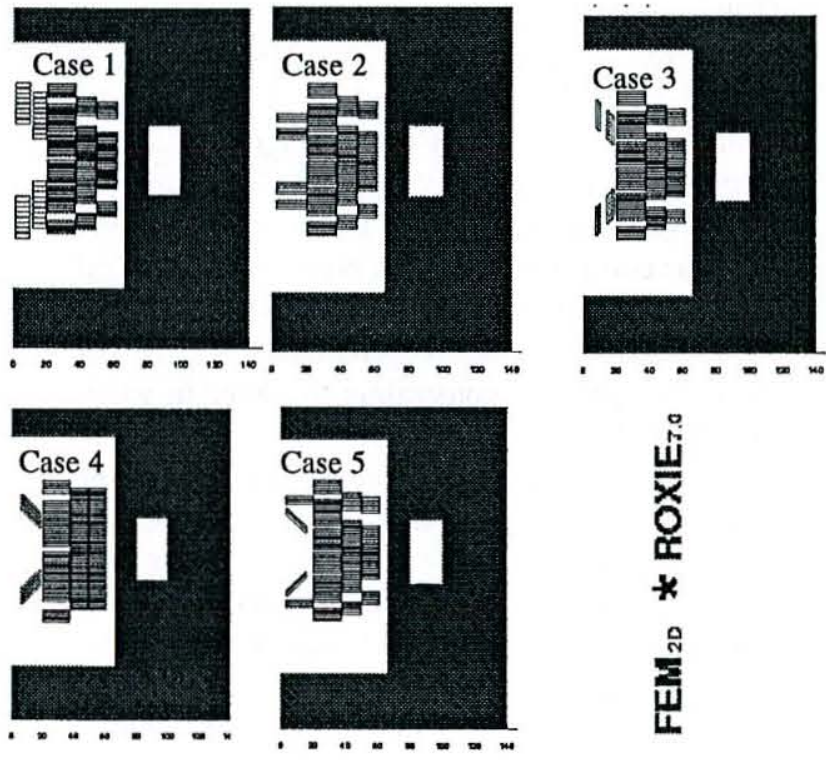

Fig. 2. Case No. 1 through 5 show a number of possible configurations of auxiliary coils that are used for minimizing field harmonics.
The case No. 5 contains two tilted turns and three straight turns. The two tilted turns play a crucial role in minimizing the higher order field harmonics. The results of preliminary investigations performed so far indicate that this geometry produces a relatively better field quality. The ends of the auxiliary coils of this case may look similar to that of a flat pancake coil (case 2, where all turns are bent easy way) if the tilt is gradually removed in a transition region between straight section and magnet end.

In this paper, the results of harmonic optimization are presented for the case No. 4 only. Table I gives the values of optimized harmonics at $1 \mathrm{kA}(1.88 \mathrm{~T})$. It may be noted that all harmonics are less than $10^{-5}$ (or tenth of a unit when expressed in the units of $10^{-4}$ ). The presence of even skew harmonics is the reflection of the missing top-bottom symmetry. In a geometry with an ideal dipole symmetry, only the odd normal harmonics will be present. Even normal and skew odd harmonics are absent because of the left-right symmetry.

TABLE I

FIELD HARMONICS AT $1 \mathrm{KA}$ (1.88 T) IN A COMMON COIL Dipole at A REFERENCE RAdIUS OF $10 \mathrm{Mm}$. AlL HARMONICS ARE LESS THAN $1 / 10$ OF A UNIT (OR $\left.10^{5}\right)$.

\begin{tabular}{|c|c|c|}
\hline $\mathrm{N}$ & SKEW $\left(\mathrm{a}_{\mathrm{n}}\right)$ & NORMAL $\left(\mathrm{b}_{\mathrm{n}}\right)$ \\
\hline 2 & 0.00 & -- \\
\hline 3 & -- & 0.00 \\
\hline 4 & -0.04 & -- \\
\hline 5 & -- & 0.00 \\
\hline 6 & 0.04 & -- \\
\hline 7 & -- & 0.01 \\
\hline 8 & 0.02 & -- \\
\hline 9 & -- & -0.07 \\
\hline 10 & 0.00 & -- \\
\hline 11 & -- & -0.05 \\
\hline 12 & 0.00 & -- \\
\hline 13 & -- & 0.04 \\
\hline 14 & 0.00 & -- \\
\hline 15 & -- & 0.01 \\
\hline
\end{tabular}

\section{B. Magnet Body-Persistent Current Induced Harmonics}

Conventional cosine theta magnets built so far with $\mathrm{Nb}_{3} \mathrm{Sn}$ superconductor have large persistent current induced harmonics [11]. A "common coil magnet system," proposed earlier [2] partly overcomes this problem. In this proposal, the return turns of the upper auxiliary coils (Fig. 1) are used to partially energize additional lower field iron-dominated aperture (upper-most aperture in Fig. 1). An outer coil generates the rest of the field in this iron-dominated aperture. The outer coil has roughly twice the current density as compared to the other coils. As in the case of McIntyre's Design [10], the persistent current induced harmonics are expected to be significantly suppressed in the two iron-dominated aperture of the 4-in-1 common coil magnet system. 
The beam is injected in this low field aperture at about 0.1 $\mathrm{T}$ and accelerated to 1.2-1.5 T. The beam is then transported to a field-matching high field aperture in a single turn transfer. Since the aperture continues to ramp-up through this operation, the problem of "snap-back" is eliminated. (The "snap-back" is referred to a sudden change in field harmonics at the beginning of an accelerating cycle). Moreover, since the beam does not stay at injection too long (as required normally for filling a machine) and since the persistent current induced harmonics are lower (relative to the central field) at higher injection field (1.2-1.5 $\mathrm{T}$ rather than 0.3-0.5 T), the beam dynamics situation improves significantly. The above approach integrates the "High Energy Booster (HEB)" in a common coil magnet system having four dipole apertures - two low field and two high field - in a single coldmass (4-in-1). This approach should result in a significant saving in the cost of building and operating a VLHC complex, with an added benefit of minimizing the problems associated with the large persistent currents in $\mathrm{Nb}_{3} \mathrm{Sn}$ magnets.

\section{Magnet Body - Iron Saturation Induced Harmonics}

Typical yoke optimization in a high field dipole involves minimizing the allowed saturation induced current dependence in harmonics while maximizing the field and minimizing the overall yoke dimensions. In addition, in the common coil magnet design, cross-talk induced even skew harmonics are also generated when the two apertures are in close proximity to minimize the overall magnet size.

The saturation-induced harmonics are minimized by adjusting the outer yoke dimensions (height and width), yoke aperture dimensions (height and width) and the saturation control holes and/or cutouts. The results of a preliminary optimization are shown in Fig. 3. All conductors are connected in series to allow using a single power supply.

The iron yoke has been optimized with ROXIE. The yoke optimization will also be carried out later using the Genetic Algorithm [12].

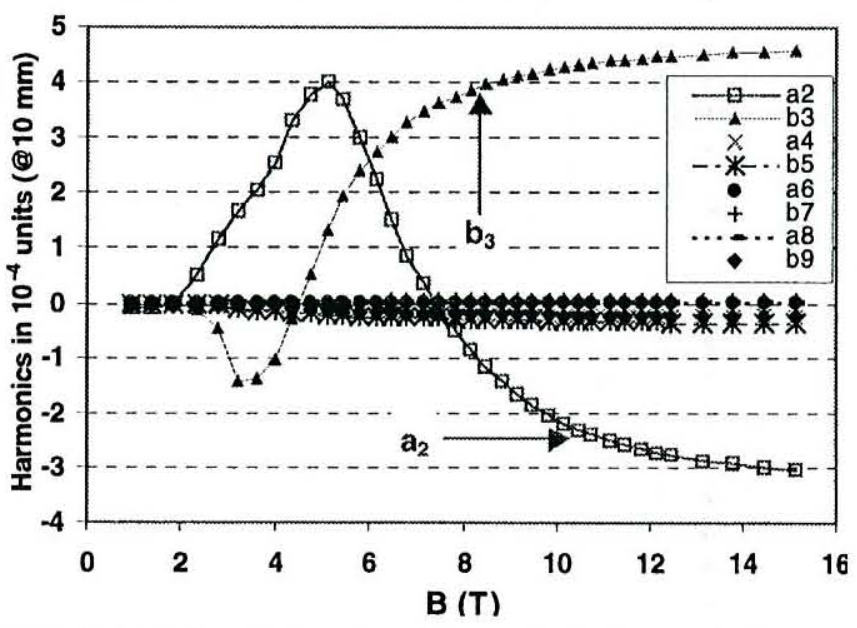

Fig. 3. Computed current dependence in harmonics due to iron saturation.

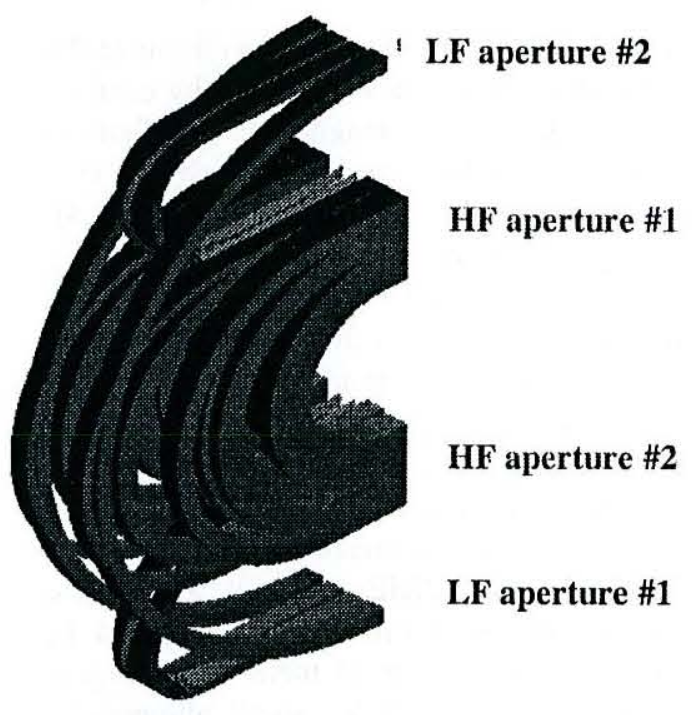

Fig 4. Optimized geometry of the return end of the common coil magnet system. The ends of the high field aperture are optimized to minimize the normal and skew harmonics.

TABLE II

INTEGRATED FIELD HARMONICS $\left(\mathrm{A}_{\mathrm{w}}, \mathrm{B}_{\mathrm{v}}\right.$ ) IN THE ENDS OF THE HIGH FIELD APERTURE OF THE COMMON COIL DIPOLE AT A REFERENCE RADIUS OF 10 MM. ALL HARMONICS ARE LESS THAN $1 / 10$ OF A UNIT $\left(10^{-5}\right)$. THE LAST TWO COLUMNS SHOW THE CONTRIBUTION OF THE TWO ENDS WHEN THE HARMONICS ARE NORMALISED FOR A 14 METER LONG DIPOLE.

\begin{tabular}{|c|c|c|c|c|}
\hline $\mathbf{n}$ & $\begin{array}{c}\mathbf{B}_{\mathbf{n}} \\
(\text { Unit-m) }\end{array}$ & $\begin{array}{c}\mathbf{A}_{\mathbf{n}} \\
\text { (Unit-m) }\end{array}$ & $\begin{array}{c}\mathbf{b}_{\mathbf{n}} \\
\text { (Units) }\end{array}$ & $\begin{array}{c}\mathbf{a}_{\mathbf{n}} \\
\text { (Units) }\end{array}$ \\
\hline $\mathbf{2}$ & 0.00 & 0.00 & 0.00 & 0.00 \\
\hline $\mathbf{3}$ & 0.01 & 0.00 & 0.00 & 0.00 \\
\hline $\mathbf{4}$ & 0.00 & -0.03 & 0.00 & 0.00 \\
\hline $\mathbf{5}$ & 0.13 & 0.00 & 0.02 & 0.00 \\
\hline $\mathbf{6}$ & 0.00 & -0.10 & 0.00 & -0.01 \\
\hline $\mathbf{7}$ & 0.17 & 0.00 & 0.02 & 0.00 \\
\hline $\mathbf{8}$ & 0.00 & -0.05 & 0.00 & -0.01 \\
\hline $\mathbf{9}$ & 0.00 & 0.00 & 0.00 & 0.00 \\
\hline $\mathbf{1 0}$ & 0.00 & -0.01 & 0.00 & 0.00 \\
\hline $\mathbf{1 1}$ & -0.01 & 0.00 & 0.00 & 0.00 \\
\hline $\mathbf{1 2}$ & 0.00 & 0.00 & 0.00 & 0.00 \\
\hline $\mathbf{1 3}$ & 0.00 & 0.00 & 0.00 & 0.00 \\
\hline
\end{tabular}

\section{Magnet Ends}

In a conventional cosine theta or a block design, the ends of a magnet primarily have a top-bottom symmetry; i.e the turns above the midplane return over the midplane and the turns below the midplane returns under in a symmetric way. Therefore, except for a small contribution from the current leads and the specific geometry of the lead end, the topbottom symmetry is maintained and thus the skew harmonics are essentially zero. The end-optimization of these designs 
involves optimizing a number of end spacers to minimize the integrated normal harmonics and peak field on the conductor. However, in a common coil magnet, the top-bottom symmetry is broken as most turns return only on one side (bottom side for upper high field aperture, see Figs. 1 and 4). This may create large skew harmonics if the ends are not properly optimized. The end spacers are strategically placed to compensate for this asymmetry so that the net conductor volume below and above the midplane is the same. The endoptimization for a common coil design requires minimization of both skew harmonics and normal field harmonics.

The geometry of an optimized coil-end is shown in Fig. 4. Table II gives the results of this optimization. The influence of iron is not included in the ROXIE model. $B_{n}$ and $A_{n}$ are the integrated normal and skew harmonics in return end. $b_{n}$ and $a_{n}$ are the net contribution to a 14 meter long magnet when normalized by the integral field. Small numbers in Table II constitutes a "Proof of Principle" that the endharmonics (both normal and skew) can be made small in a common coil magnet.

It should be pointed out that the axial component of the field $\left(B_{z}\right)$ is non-zero in each of the two common coil end. In a $15 \mathrm{~T}$ magnet, the value can be as large as 1 Tesla-meter. However, the sign of this field is opposite in the two ends (return end and lead end). Therefore, the end field of one magnet would cancel the end field of the next nearby magnet and the integral $\mathrm{B}_{\mathrm{z}}$ field of each magnet will also be zero. The influence of this on beam dynamics still needs to be examined.

\section{SUMMARY OF THE DESIGN}

A few important parameters of an initially optimized design are given in Table III. The expected quench field of this design is $14.8 \mathrm{~T}$. There has been continuous improvement in $\mathrm{Nb}_{3} \mathrm{Sn}$ performance [13] and a better $\mathrm{J}_{\mathrm{c}}$ than the one used here $\left(2000 \mathrm{~A} / \mathrm{mm}^{2}\right)$ is available today.

A term, "Average number of coils (layers) per half aperture," has been introduced in Table III. This facilitates a comparison of the number of coils per aperture between 2-in1 common coil design with 2-in-1 (or single aperture) cosine theta or block designs. There are 14 coils in the 4 aperture magnet and hence the number 1.75 per half-aperture. If the iron-dominated low field apertures are not to be utilized then only 12 coils will be required for two apertures and hence the number 3 per half-aperture in Table III.

\section{CONCLUSIONS}

The results of initial field quality optimization show that a good geometric field quality (with harmonics in $10^{-5}$ range), can be obtained in a common coil magnet design. The same design has a small saturation induced harmonics. It is also shown that the end geometry can be optimized for small skew and normal harmonics.
TABLE III

PARAMETERS OF AN OPTIMIZED COMMON COIL MAGNET DESIGN. HF INDICATES THE HIGH FIELD APERTURE AND LF THE LOW FIELD.

\begin{tabular}{|l|l|}
\hline Coil aperture (HF) & $40 \mathrm{~mm}$ \\
\hline Coil aperture (LF), Horizontal X Vertical & $40 \mathrm{~mm} \mathrm{X} \mathrm{20} \mathrm{mm}$ \\
\hline Avg. No. of coils per half aperture (4-in-1) & 1.5 \\
\hline Avg. No. of coils per half aperture (2-in-1) & 2.5 \\
\hline Number of layers & $3+1$ \\
\hline Computed quench field at 4.2 K & $14.8 \mathrm{~T}$ \\
\hline Peak Fields, inner \& outer layers & $15.0 \mathrm{~T} \mathrm{\&} 10.5 \mathrm{~T}$ \\
\hline Quench current & $12.1 \mathrm{kA}$ \\
\hline Wire Non-Cu J sc $4.2 \mathrm{~K}, 12 \mathrm{~T}$ ) & $2000 \mathrm{~A} / \mathrm{mm}^{2}$ \\
\hline Strand diameter & $0.8 \mathrm{~mm}$ \\
\hline No. of strands, inner \& outer layers & 40,26 \\
\hline Cable width, inner \& outer layer (insulated) & $16.9 \mathrm{~mm}, 11.1 \mathrm{~mm}$ \\
\hline Cu/Non-Cu ratio, inner \& outer & $0.7,1.7$ \\
\hline No. of turns per quadrant per aperture (HF) & 80 \\
\hline Max. height of each layer from midplane & $40 \mathrm{~mm}$ \\
\hline Bore spacing (between HF) & $220 \mathrm{~mm}$ \\
\hline Minimum coil bend radius (in ends) & $70 \mathrm{~mm}$ \\
\hline Yoke size (full width X full height) & $280 \mathrm{~mm} \mathrm{X} \mathrm{600} \mathrm{mm}$ \\
\hline
\end{tabular}

\section{ACKNOWLEDGEMENT}

Discussions with the other members of the SUPERCON Group at the Lawrence Berkeley National Laboratory are highly appreciated.

\section{REFERENCES}

[1] R. Gupta, "A Common Coil Design for High Field 2-in-1 Accelerator Magnets," Proceedings of the 1997 Particle Accelerator Conference.

[2] R. Gupta, "Common Coil Magnet System for VLHC," Proceedings of the 1999 Particle Accelerator Conference.

[3] R. Gupta, et al., "A High Field Magnet Design for a Future Hadron Collider," Proceedings of the 1998 Applied Superconductivity Conference, Palm Desert, CA, Sept. 1998.

[4] W. Sampson, et al., "A Common Coil Magnet for Testing High Field Superconductors," Procd. of the 1999 Particle Accel. Conference.

[5] S. Gourlay, et al., "Design and Fabrication of A $14 \mathrm{~T} \mathrm{Nb}_{3} \mathrm{Sn}$ Superconducting Racetrack Dipole Magnet," these proceedings.

[6] G. Sabbi, et al., "Conceptual Design of a Common Coil Dipole for VLHC," these proceedings.

[7] S. Russenschuck, "ROXIE - A Computer Code for the Integrated Design of Accelerator Magnets," Sixth European Particle Accelerator Conference, Stockholm, Sweden, June 1998.

[8] K. Preis, et al., "Numerical Analysis of 3d Magneto-static Fields, "IEEE Transactions on Magnetics," 27, 3798 - 3803, 1991.

[9] VLHC Web site: http://vlhc.org/.

[10] P. McIntyre, et al., "Optimized Block-Coil Dipoles For Future Hadron Colliders," these proceedings.

[11] A. McInturff, et al., "Operational Characteristic, Parameters, and History of a (13T) $\mathrm{Nb}_{3} \mathrm{Sn}$ Dipole," Proceedings of the 1999 Particle Accelerator Conference.

[12] S. Ramberger and S. Russenschuck, "Genetic Algorithm with Niching for Conceptual Design Studies," IEEE Trans. on Mag., 34(5): 1998.

[13] R. Scanlan, "Conductor Development for High Field Dipole Magnets," these proceedings. 